\title{
A Note on Best Proximity Point Theorems under Weak $P$-Property
}

\author{
Angel Almeida, ${ }^{1}$ Erdal Karapınar, ${ }^{2,3}$ and Kishin Sadarangani ${ }^{1}$ \\ ${ }^{1}$ Departamento de Matemáticas, Universidad de Las Palmas de Gran Canaria, Campus de Tafira Baja, \\ 35017 Las Palmas de Gran Canaria, Spain \\ ${ }^{2}$ Department of Mathematics, Atilim University, Incek, 06836 Ankara, Turkey \\ ${ }^{3}$ Nonlinear Analysis and Applied Mathematics Research Group (NAAM), King Abdulaziz University, \\ Jeddah, Saudi Arabia
}

Correspondence should be addressed to Erdal Karapınar; erdalkarapinar@yahoo.com

Received 15 November 2013; Accepted 9 February 2014; Published 12 March 2014

Academic Editor: Mohamed Boussairi Jleli

Copyright (C) 2014 Angel Almeida et al. This is an open access article distributed under the Creative Commons Attribution License, which permits unrestricted use, distribution, and reproduction in any medium, provided the original work is properly cited.

In the very recent paper of Akbar and Gabeleh (2013), by using the notion of $P$-property, it was proved that some late results about the existence and uniqueness of best proximity points can be obtained from the versions of associated existing results in the fixed point theory. Along the same line, in this paper, we prove that these results can be obtained under a weaker condition, namely, weak $P$-property.

\section{Introduction and Preliminaries}

Let $A$ and $B$ be two nonempty subsets of a metric space $(X, d)$. By $A_{0}$ and $B_{0}$, we denote the following sets:

$$
\begin{aligned}
& A_{0}=\{x \in A: d(x, y)=d(A, B) \text { for some } y \in B\}, \\
& B_{0}=\{y \in B: d(x, y)=d(A, B) \text { for some } x \in A\}, \\
& \text { where } d(A, B)=\inf \{d(x, y): x \in A \text { and } y \in B\} .
\end{aligned}
$$

Definition 1. Let $A$ and $B$ be a pair of nonempty subsets of a metric space $(X, d)$ and let $T: A \rightarrow B$ be a mapping. One will say that $x^{*} \in A$ is a best proximity of $T$ if

$$
d\left(x^{*}, T x^{*}\right)=d(A, B) .
$$
follows.

In [1], the authors introduced the notion of $P$-property as

Definition 2 (see [1]). Let $(A, B)$ be a pair of nonempty subsets of a metric space $(X, d)$ with $A_{0} \neq \emptyset$. Then, the pair $(A, B)$ is said to have the $P$-property if

$$
\left.\begin{array}{l}
d\left(x_{1}, y_{1}\right)=d(A, B) \\
d\left(x_{2}, y_{2}\right)=d(A, B)
\end{array}\right\} \Longrightarrow d\left(x_{1}, x_{2}\right)=d\left(y_{1}, y_{2}\right)
$$

where $x_{1}, x_{2} \in A_{0}$ and $y_{1}, y_{2} \in B_{0}$.
Very recently, Zhang et al. [2] introduced the weak $P$ property.

Definition 3 (see [2]). Let $(A, B)$ be a pair of nonempty subsets of a metric space $(X, d)$ with $A_{0} \neq \emptyset$. Then, the pair $(A, B)$ is said to have the $P$-property if

$$
\left.\begin{array}{l}
d\left(x_{1}, y_{1}\right)=d(A, B) \\
d\left(x_{2}, y_{2}\right)=d(A, B)
\end{array}\right\} \Longrightarrow d\left(x_{1}, x_{2}\right) \leq d\left(y_{1}, y_{2}\right)
$$

where $x_{1}, x_{2} \in A_{0}$ and $y_{1}, y_{2} \in B_{0}$.

It is evident that if a pair $(A, B)$ has the $P$-property, then it has the weak $P$-property. In [2], the authors established some examples to prove that the converse of the statement above is false.

In the literature, a great number of fixed point theorems have appeared to generalize, extend, and improve the celebrated Banach's contraction principle. Among them, we present two of these results as examples and we prove that their versions in the context of the best proximity point theory can be deduced as consequences of results in the setting of fixed point theory.

Let $\Phi$ denote the set of all functions $\phi:[0, \infty) \rightarrow[0, \infty)$ which satisfy 
$\left(\phi_{1}\right) \phi$ is continuous, and nondecreasing;

$\left(\phi_{2}\right) \phi$ is positive on $(0, \infty)$ and $\phi(0)=0$.

Theorem 4 (see [3]). Let $(X, d)$ be a complete metric space and $T: X \rightarrow X$ an operator satisfying

$$
d(T x, T y) \leq d(x, y)-\phi(d(x, y)) \quad \text { for any } x, y \in X
$$

where $\phi \in \Phi$ which satisfy $\lim _{t \rightarrow \infty} \phi(t)=\infty$. Then, $T$ has a unique fixed point.

Remark 5. Notice that Theorem 4 remains valid if we remove the assumption that $\lim _{t \rightarrow \infty} \phi(t)=\infty$.

Definition 6. Let $\Psi$ denote the family of all functions $\psi$ : $[0,+\infty) \rightarrow[0,+\infty)$ which satisfy

$\left(\psi_{1}\right) \lim _{n \rightarrow \infty} \psi^{n}(t)=0$ for each $t>0$, where $\psi^{n}$ is the $n$th iterate of $\psi$.

$\left(\psi_{2}\right) \psi$ is nondecreasing.

Remark 7. A function, $\psi \in \Psi$, is known as a comparison function or Bianchini-Grandolfi gauge function in the literature [4]. Moreover, it is easily seen that such functions satisfy the conditions

$$
\psi(t)<t \quad \text { for } t>0, \psi(0)=0 \text {. }
$$

In 2012, Romaguera [5] proved the analog of the following fixed point theorem in the context of partial metric spaces. It is evident that its metric version remains true.

Theorem 8 (see [5]). Let $(X, d)$ be a complete metric space and $T: X \rightarrow X$ an operator satisfying

$$
d(T x, T y) \leq \psi\left(M_{T}(x, y)\right) \quad \text { for any } x, y \in X
$$

where $\psi \in \Psi$ and

$$
M_{T}(x, y)=\max \{d(x, y), d(x, T x), d(y, T y)\} .
$$

Then, T has a unique fixed point.

\section{Main Results}

We start this section with the following lemma which appeared implicitly in [2]. We prove this lemma for that the paper is self-contained.

Lemma 9. Let $(A, B)$ be a pair of nonempty closed subsets of a complete metric space $(X, d)$. Suppose that the following conditions hold:

(i) $A_{0} \neq \emptyset$;

(ii) the pair $(A, B)$ has the weak P-property.

Then, the set $B_{0}$ is closed.
Proof. We have $B_{0} \neq \emptyset$ due to the assumption that $A_{0} \neq \emptyset$. Let $\left(y_{n}\right) \subset B_{0}$ be a sequence such that $y_{n} \rightarrow y \in B$. We shall prove that $y \in B_{0}$. In fact, since $\left(y_{n}\right) \subset B_{0}$, we find a sequence $\left(x_{n}\right) \subset A_{0}$ such that

$$
d\left(x_{n}, y_{n}\right)=d(A, B) \quad \text { for any } n \in \mathbb{N} .
$$

By using the weak $P$-property, we infer that

$$
d\left(x_{n}, x_{m}\right) \leq d\left(y_{n}, y_{m}\right) \quad \text { for any } n, m \in \mathbb{N} .
$$

As $\left(y_{n}\right)$ is a Cauchy sequence in $B$; from the inequality (10), we derive that the sequence $\left(x_{n}\right)$ is a Cauchy sequence in $A$. Therefore, since $A$ is closed, we deduce that $x_{n} \rightarrow x \in A$ for certain $x \in A$.

Finally, by the continuity of the metric $d$ together with (9), it gives us $d(x, y)=d(A, B)$. Hence, $y \in B_{0}$.

Lemma 10. Let $(A, B)$ be a pair of nonempty closed subsets of a complete metric space $(X, d)$. Suppose that the following conditions hold:

(i) $A_{0} \neq \emptyset$;

(ii) the pair $(A, B)$ has the weak P-property;

(iii) $T: A \rightarrow B$ is a continuous mapping with $T\left(A_{0}\right) \subset B_{0}$. Then, we have $T\left(\overline{A_{0}}\right) \subset B_{0}$.

Proof. It is sufficient to prove that if $x \in \overline{A_{0}} \backslash A_{0}$, then $T x \in B_{0}$. In fact, since $x \in \overline{A_{0}} \backslash A_{0}$, we can find a sequence $\left(x_{n}\right) \subset A_{0}$ such that $x_{n} \rightarrow x$. Due to (iii), we have $T\left(x_{n}\right) \subset B_{0}$. Since the mapping $T$ is continuous and $B_{0}$ is closed by Lemma 9 , we conclude that $T x \in B_{0}$.

Before stating the main result of this paper, we need to recall the main result of Sankar Raj [1].

Theorem 11. Let $(A, B)$ be a pair of nonempty closed subsets of a complete metric space $(X, d)$ with $A_{0} \neq \emptyset$. Let $T: A \rightarrow B$ be a mapping satisfying

$$
d(T x, T y) \leq d(x, y)-\phi(d(x, y)) \quad \text { for any } x, y \in X \text {, }
$$

where $\phi \in \Phi$. Suppose also that the pair $(A, B)$ has the $P$ property and $T\left(A_{0}\right) \subset B_{0}$. Then, $T$ has a unique best proximity point.

Now, we present the main results of the paper. If in Theorem 11 we replace $P$-property by the weak $P$-property, we derive the following result.

Theorem 12. Theorem 11 under the the assumption of the weak $P$-property instead of the P-property is a consequence of Theorem 4.

Proof. Suppose that all the conditions of Theorem 11 are fulfilled under the the assumption of the weak $P$-property 
instead of the $P$-property. We first note that $T$ is continuous, since

$$
d(T x, T y) \leq d(x, y)-\phi(d(x, y)) \leq d(x, y)
$$

for any $x, y \in X$. Hence, by Lemma 10 , we have $T\left(\overline{A_{0}}\right) \subset B_{0}$.

Next, we define an operator $P: T\left(\overline{A_{0}}\right) \rightarrow A_{0}$ by $P y=x$ such that $d(x, y)=d(A, B)$. As $y \in T\left(\overline{A_{0}}\right) \subset B_{0}$, we can find $x \in A_{0}$ such that $d(x, y)=d(A, B)$. Moreover, $P$ is a welldefined mapping. Suppose, on the contrary, that there exists another $x_{0} \in A_{0}$ such that $d\left(x_{0}, y\right)=d(A, B)$. By using the fact that the pair $(A, B)$ has the weak $P$-property, we derive that

$$
d\left(x_{0}, x\right) \leq d(y, y)=0,
$$

and, consequently, $x=x_{0}$.

Now, we consider the operator $P \circ T: \overline{A_{0}} \rightarrow A_{0}$. In the sequel, we shall prove that the operator $P \circ T$ satisfies all assumptions of Theorem 4 . Notice that $\left(\overline{A_{0}}, d\right)$ is a complete metric space since $\overline{A_{0}}$ is closed.

On the other hand, we have

$$
\begin{aligned}
& d(P(T x), T x)=d(A, B) \\
& d(P(T y), T y)=d(A, B),
\end{aligned}
$$

for $x, y \in \overline{A_{0}}$. As the pair $(A, B)$ has the weak $P$-property, we infer from (14) that

$$
d(P(T x), P T y) \leq d(T x, T y) .
$$

Regarding (11), we derive that

$$
d(P(T x), P T y) \leq d(T x, T y) \leq d(x, y)-\phi(d(x, y)),
$$

for any $x, y \in \overline{A_{0}}$.

Therefore, by Theorem 4 , there exists a unique fixed point $x^{*} \in \overline{A_{0}}$ of $P \circ T$, that is, $P\left(T x^{*}\right)=x^{*}$. By definition, we have

$$
d\left(x^{*}, T x^{*}\right)=d(A, B) .
$$

Therefore, $x^{*}$ is a best proximity point of the mapping $T$ and this completes the existence part of the proof.

We shall show that $x^{*}$ is the unique best proximity of the mapping T. Suppose, on the contrary, that $z^{*}$ is another best proximity of the mapping $T$. Consequently, we have

$$
d\left(z^{*}, T z^{*}\right)=d(A, B),
$$

and also $z^{*} \in A_{0} \subset \overline{A_{0}}$. Moreover, taking into account the definition of the operator $P$, this means that

$$
P\left(T z^{*}\right)=z^{*} \text {. }
$$

Hence, $z^{*}$ is a fixed point of $P \circ T$. By the uniqueness of the fixed point of $P \circ T$, we deduce that $x^{*}=z^{*}$, which completes the proof.

Theorem 4 imposes Theorem 11 because we have used a weaker condition, namely, the notion of the weak $P$-property.

Theorem 13. Let $(A, B)$ be a pair of nonempty closed subsets of a complete metric space $(X, d)$ with $A_{0} \neq \emptyset$. Let $T: A \rightarrow B$ be a continuous mapping satisfying

$$
d(T x, T y) \leq \psi\left(M_{T}(x, y)\right) \quad \text { for any } x, y \in X,
$$

where $\psi \in \Psi$ and

$$
M_{T}(x, y)=\max \{d(x, y), d(x, T x), d(y, T y)\} .
$$

Suppose also that the pair $(A, B)$ has the weak P-property and $T\left(A_{0}\right) \subset B_{0}$. Then, $T$ has a unique best proximity point.

By using the same techniques used in Theorem 12, we derive Theorem 13.

Theorem 14. Theorem 13 is a consequence of Theorem 8.

To avoid the repetition, we omit the proof Theorem 14.

Remark 15. Notice that in Theorem 14, we assume that $T$ is continuous since the contractive condition (20) appeared in Theorem 13 does not imply the continuity of $T$.

Corollary 16. Let $(A, B)$ be a pair of nonempty closed subsets of a complete metric space $(X, d)$ with $A_{0} \neq \emptyset$. Let $T: A \rightarrow B$ be a continuous mapping satisfying

$$
d(T x, T y) \leq \psi\left(M_{T}(x, y, A, B)\right) \quad \text { for any } x, y \in X,
$$

where $\psi \in \Psi$ and

$$
\begin{gathered}
M_{T}(x, y, A, B)=\max \{d(x, y), d(x, T x)-d(A, B), \\
d(y, T y)-d(A, B)\} .
\end{gathered}
$$

Suppose also that the pair $(A, B)$ has the weak P-property and $T\left(A_{0}\right) \subset B_{0}$. Then, $T$ has a unique best proximity point.

We skip the proof since the mapping $\psi$ is nondecreasing and hence we get

$$
\begin{array}{r}
d(T x, T y) \leq \psi\left(M_{T}(x, y, A, B)\right) \leq \psi\left(M_{T}(x, y)\right) \\
\text { for any } x, y \in X .
\end{array}
$$

Therefore, Corollary 16 is a consequence of Theorem 13.

Remark 17. Corollary 16 can be considered as a partial version of Theorem 20 of [6].

\section{Conflict of Interests}

The authors declare that they have no conflict of interests.

\section{Authors' Contribution}

All authors contributed equally and significantly in writing this paper. All authors read and approved the final paper.

\section{Acknowledgment}

The authors are grateful to the reviewers for their careful reviews and useful comments.

\section{References}

[1] V. Sankar Raj, "A best proximity point theorem for weakly contractive non-self-mappings," Nonlinear Analysis; Theory, Methods \& Applications, vol. 74, no. 14, pp. 4804-4808, 2011. 
[2] J. Zhang, Y. Su, and Q. Cheng, "A note on 'a best proximity point theorem for Geraghty-contractions"' Fixed Point Theory and Applications, vol. 2013, article 99, 2013.

[3] B. E. Rhoades, "Some theorems on weakly contractive maps," Nonlinear Analysis; Theory Methods \& Applications, vol. 47, no. 4, pp. 2683-2693, 2001.

[4] I. A. Rus, Generalized Contractions and Applications, Cluj University Press, Cluj-Napoca, Romania, 2001.

[5] S. Romaguera, "Fixed point theorems for generalized contractions on partial metric spaces," Topology and Its Applications, vol. 159, no. 1, pp. 194-199, 2012.

[6] M. Jleli, E. Karapinar, and B. Samet, "On best proximity points under the $P$-property on partially ordered metric spaces," Abstract and Applied Analysis, vol. 2013, Article ID 150970, 6 pages, 2013. 


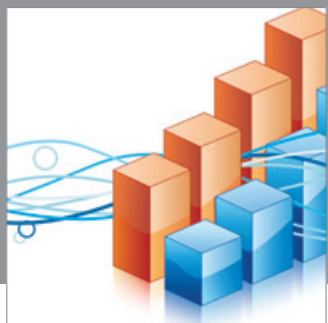

Advances in

Operations Research

mansans

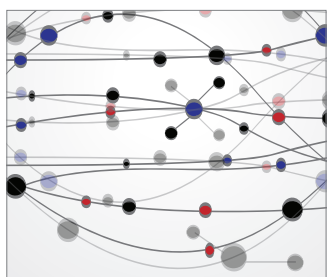

The Scientific World Journal
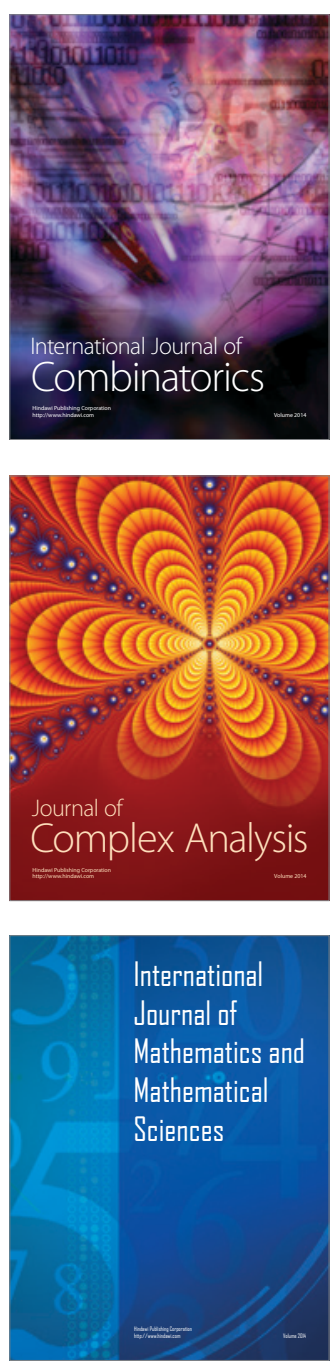
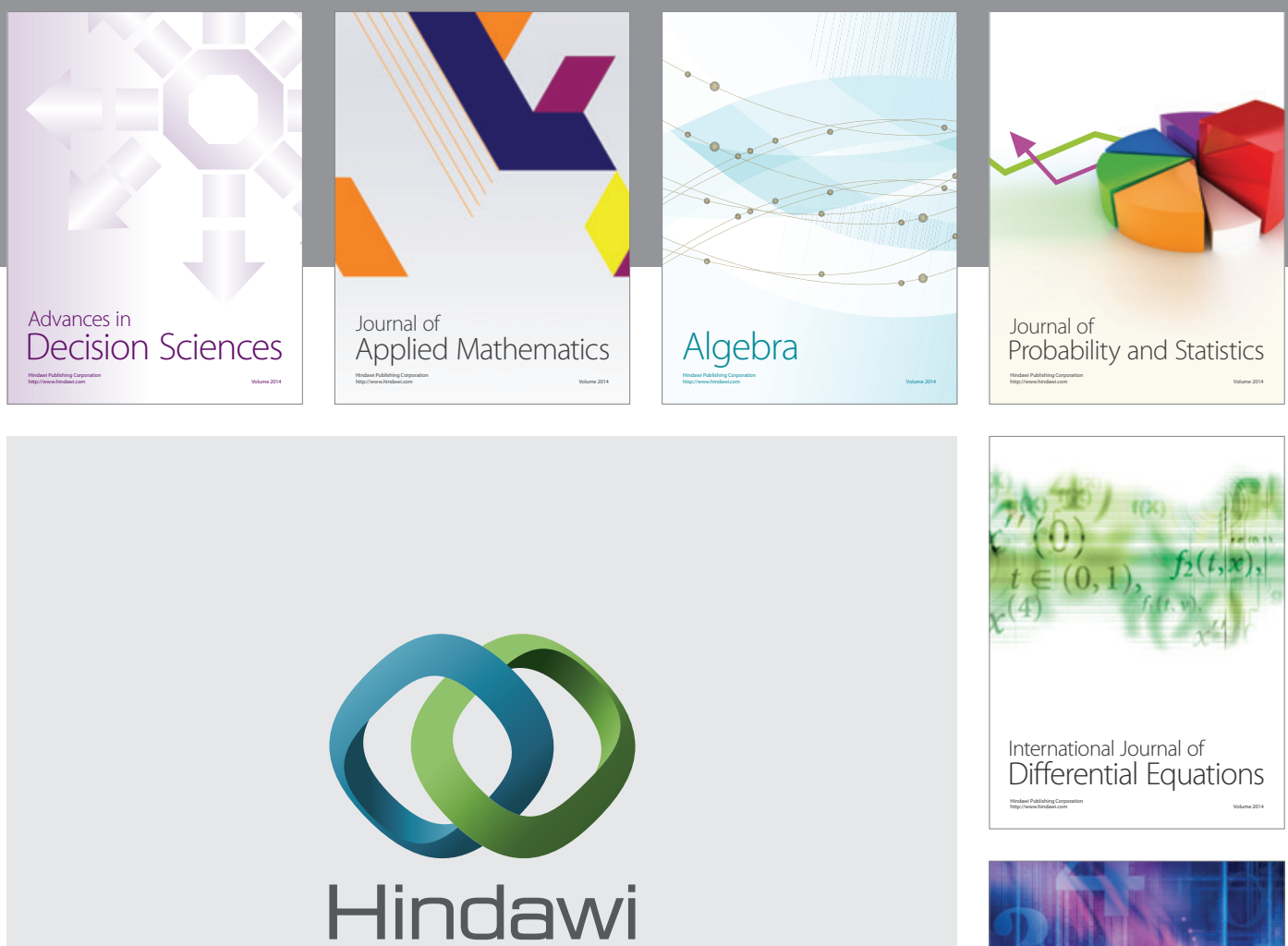

Submit your manuscripts at http://www.hindawi.com
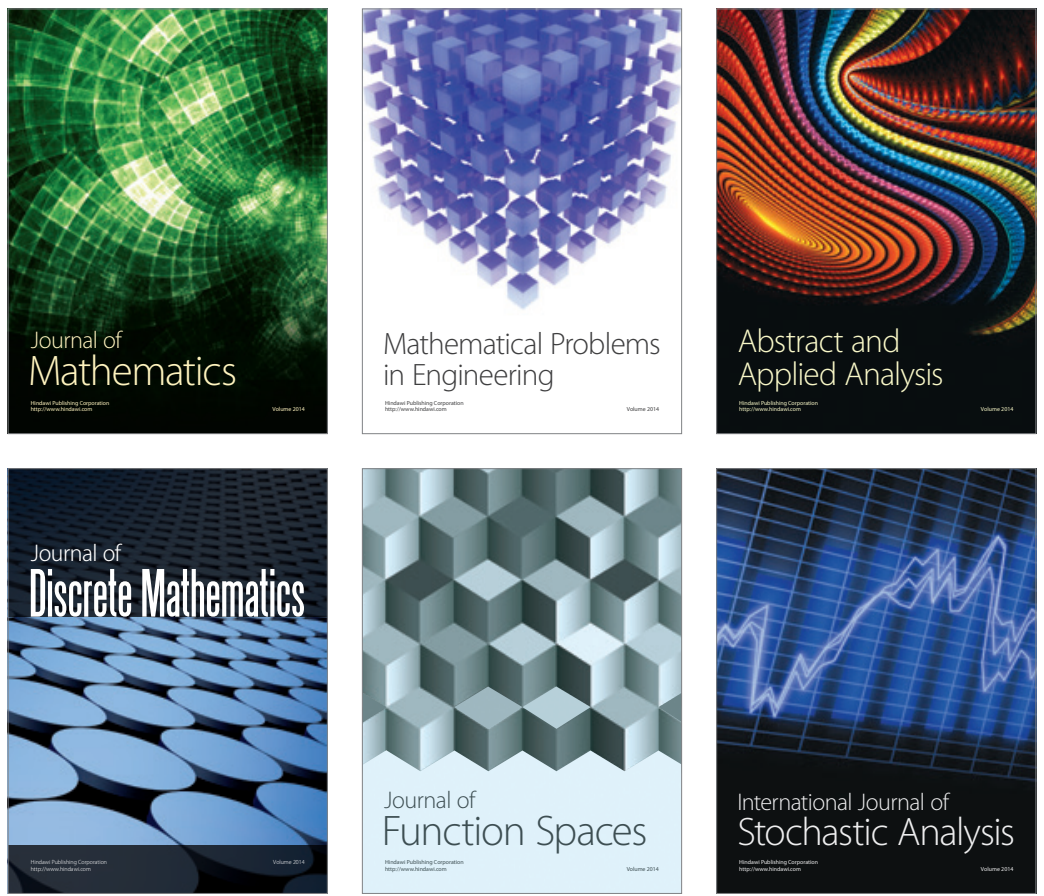

Journal of

Function Spaces

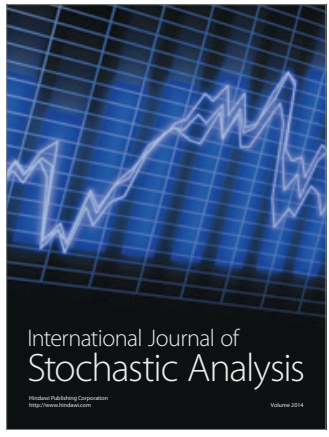

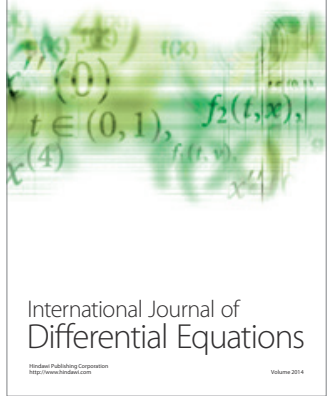
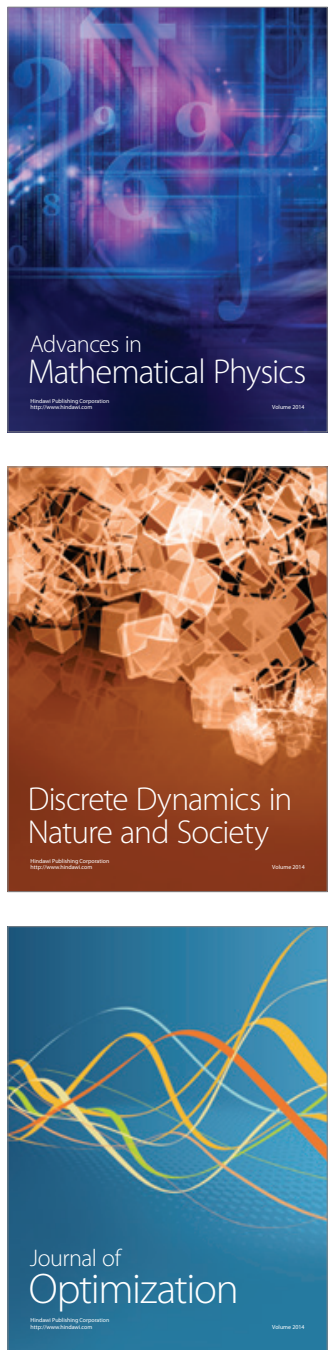